\title{
Realidad aumentada como recurso educativo en materias administrativas de alumnos de diseño industrial
}

\section{Augmented Reality as an educational resource in administrative matters for industrial design students}

\author{
SOBERANES-MARTÍN, Anabelem†**, CASTILLO MENDOZA, José Luis y PEÑA MARTÍN, Aideé \\ Universidad Autónoma del Estado de México, Centro Universitario UAEM Valle de Chalco, Hermenegildo Galeana \\ \#3, Col. María Isabel, Valle de Chalco Solidaridad, Estado de México. C. P. 56615
}

ID $1^{\mathrm{er}}$ Autor: Anabelem, Soberanes-Martín / ORC ID: 0000-0002-1101-8279, Research Gate ID: Anabelem_Martin, CVU CONACYT ID: 180105.

ID $1^{\mathrm{er}}$ Coautor: José Luis, Castillo-Mendoza / ORC ID: 0000-0002-5668-0602, Researcher ID: S-8059-2018, CVU CONACYT ID: 898715.

ID $2^{\text {do }}$ Coautor: Aideé Peña Martín / ORC ID: 0000-0003-2688-2155, Researcher ID: S-8069-2018, CVU CONACYT ID: 947584

DOI: $10.35429 / J A D .2019 .7 .3 .11 .12$

Recibido 12 de Enero, 2019; Aceptado 28 de Marzo, 2019

\begin{abstract}
Resumen
La realidad aumentada va en auge en diversas áreas, la educación no es la excepción, una de las asignaturas que a veces es un tanto compleja para los alumnos de diseño industrial es Mercado y Consumo, motivo por el cual se planteó como objetivo desarrollar una aplicación móvil con realidad aumentada que, en una primera fase incluya los aspectos teóricos de la materia, después se incluyan videos y animaciones, que presente la información de refuerzo e integración de ejercicios de algunos conceptos incorporando diversos recursos. Se identifican tres etapas: 1) aspectos generales, se establecieron los participantes del estudio, los materiales a desarrollar, se seleccionaron los instrumentos que se aplicarían para determinar la calidad de la aplicación, 2) se seleccionó para su desarrollo el software Unity, para ser utilizado en ambas plataformas IOS y Android; 3) se aplicó una rúbrica sobre la calidad de aplicaciones móviles educativas a 9 docentes y 64 estudiantes elegidos de manera aleatoria de la licenciatura en diseño industrial, algunos de los resultados fueron $94.7 \%$ y $99.6 \%$ de aceptación de la aplicación por los profesores y alumnos respectivamente.
\end{abstract}

Realidad aumentada, Recurso educativo, Diseñador industrial

\begin{abstract}
Augmented reality is booming in several areas, education is no exception, one of the subjects that is sometimes somewhat complicated for students of industrial design is Market and Consumption, which is why it was proposed as an objective to develop a mobile application with augmented reality that in a first phase includes the theoretical aspects of the subject, then present videos and animations, presenting information reinforcement and integration of exercises of some concepts incorporating various resources. Three stages were identified: 1) general aspects, the participants of the study were established, the materials to be developed, the instruments that would be applied to determine the quality of the application were selected, 2) the Unity software was selected for its development, for both IOS and Android platforms; 3) a rubric on the quality of mobile educational applications was applied to 9 teachers and 64 students randomly chosen from the degree in industrial design, some of the results were $94.7 \%$ and $99.6 \%$ of acceptance of the application by teachers and students respectively.
\end{abstract}

Augmented reality, Educational resource, Industrial designer

Citación: SOBERANES-MARTÍN, Anabelem, CASTILLO MENDOZA, José Luis y PEÑA MARTÍN, Aideé. Realidad aumentada como recurso educativo en materias administrativas de alumnos de diseño industrial. Revista de Arquitectura y Diseño. 2019. 3-7: 11-21.

*Correspondencia al Autor (Correo Electrónico: asoberanesm@uaemex.mx)

$\dagger$ Investigador contribuyendo como primer autor. 


\section{Introducción}

La Licenciatura en Diseño Industrial (LDI) es una disciplina orientada a la creación de productos, físicos o abstractos, que solucionen alguna necesidad del cliente o usuario para mejorar la funcionalidad y estética de un servicio o producto; por lo que se enfoca a plasmar bocetos que pueden ser trazados en diferentes bosquejos con tendencia más artística; es una actividad multidisciplinaria e inventiva (UAMéx, 2015). Los alumnos que ingresan esperan cursar materias con este enfoque; sin embargo, el plan de estudios comprende áreas de administración, matemáticas, mercadotecnia, procesamiento de materiales y tecnología, lo cual en algunos casos les caso problema para aprender de esas temáticas. De manera que un punto de partida sería identificar el estilo de aprendizaje para que ayudé a los docentes y al mismo alumno definir de manera estrategias de aprendizaje acordes a cada área (Castillo et al., 2016a).

El área económica administrativa del plan de estudios de LDI tiene por objetivo "Fundamentar las propuestas de diseño industrial desde la perspectiva de la gestión estratégica empresarial mediante el análisis de los factores económicos, de mercado y de negociación que impacta los ámbitos local, regional y global [...]" (UAEMéx, 2015, p. 186). En 2016 se aplicó una prueba para identificar los estilos de aprendizaje del LDI, en cuanto a la preferencia del estilo de aprendizaje $35.7 \%$ son visuales, $33.9 \%$ kinestésicos y $15.8 \%$ auditivos (Castillo et al., 2016a).

Por ello, los docentes buscan incorporar herramientas que permiten apoyar los diversos estilos de aprendizaje de los LDI, se considera lo que expresan Cabero-Almenara, De la Horra, \& Sánchez (2018), un gran número de tecnologías está acercándose a las instituciones educativas.

De acuerdo con Martins et al. (2019) la Realidad Aumentada (RA) y la Realidad Virtual (RV) "[...] utilizan diversos canales y soportes que favorecen la creación de ambientes adecuados para los diferentes estilos de aprendizajes [...], favoreciendo una mayor capacidad de percepción, de razonamiento abstracto y espacial."
El uso de la RA es cada vez más frecuente, permite a un individuo interactuar con su propio entorno mediante un dispositivo digital, lo ayuda a observar un entorno más extenso y diverso en cualquier ámbito (Arteaga \& Pino, 2018). En el caso de la RA, la constante actualización de los dispositivos propicia que exista una mayor relación usuariointerfaz y permite que más personas tengan acceso a las tecnologías, que aumenten y enriquezcan la vida profesional, académica y cotidiana (Badilla \& Sandoval, 2015). De igual manera, Arteaga \& Pino (2018) señalan que la RA se está introduciendo en diversas áreas de la educación, que permite desarrollar habilidades innatas, en la formación del individuo durante su proceso de vida educacional. Confirmando estas posturas, Cabero-Almenara, VázquezCano \& López-Meneses (2018) expresan "la realidad aumentada puede ayudar a incrementar el valor de otras tecnologías en el aspecto formativo y práctico del desarrollo de las competencias profesionales y académicas del alumnado universitario." (p. 29).

La RA hace uso de dispositivos móviles, en un estudio realizado por LagunesDomínguez et al. (2017) identifican que los estudiantes cuentan con los recursos tecnológicos para el aprendizaje móvil, en su estudio se identificó un porcentaje mayor a 98\%; además, con un porcentaje de $92.65 \%$ de aceptación para que dispositivos móviles sean incorporados en clase, también de no existir diferencia si los estudiantes son del área de administración o de sistemas.

Pero como toda tecnología, tiene beneficios y algunas limitantes; entre los beneficios de la RA en la educación está "captar la atención del grupo objetivo motivándolo a participar de manera más activa en las experiencias de clases totalmente presenciales tradicionales, así como también para aquellas que se realizan totalmente a distancia." (Bernal \& Ballesteros-Ricaurte, 2017, p. 9). Cheng (2017) hace alusión a la RA destacando al mismo tiempo que su uso fomenta actitudes positivas hacia el aprendizaje. Algunas investigaciones (DíazCampos, 2016; Prendes, 2015) señala que la realidad aumentada es una tecnología que puede ayudar a mejorar el proceso de enseñanza-aprendizaje. 
Asimismo, la RA logra un mayor acceso a la información, genera marcos innovadores de actividad, como: determinados grupos virtuales, interacciones gratificantes, nuevas escalas de valores, entre otros (Fombona, PascualSevillano, \& González-Videgaray, 2017). También, la RA viene a sustituir la experiencia de las visitas de campo al poder realizarse en el propio dispositivo (Harley et al., 2016).

Algunos autores (Villalustre \& Del Moral, 2018; León, Duque \& Escobar, 2018) señalan que la experiencia en las aulas universitarias de formación ha resultado una práctica innovadora, motivadora y sirve de apoyo a los docentes para potenciar su función.

El Tecnológico de Monterrey (2017, p. 13) indica que las mejores prácticas responden a un enfoque pedagógico orientado al aprendizaje activo (learning by doing), al ser los alumnos quienes deciden cómo combinar la información aumentada. Cabero, Barroso \& Llorente (2019) expresan que los alumnos muestran altos niveles de satisfacción cuando participan en experiencias de RA, independiente del nivel de estudios o de contenidos curriculares sobre los que trate.

Además, los estudiantes consideran que "sería interesante su uso en diferentes contextos formativos como un recurso que promueve la creatividad, la imaginación y la curiosidad de la persona, además de construir nuevos conocimientos e interaccionar de una manera tan directa con lo virtual." (Cabero-Almenara, Vázquez-Cano \& López-Meneses, 2018, p. 29). León, Duque y Escobar (2018) afirman "que los estudiantes son capaces de desarrollar competencias basadas en el descubrimiento, la investigación y la construcción del conocimiento de una forma dinámica, creativa y reflexiva utilizando realidad aumentada." (p. 812). Cabero-Almenara, Vázquez-Cano \& López-Meneses (2018) expresan que la experiencia didáctica de aplicación de la RA con alumnado universitario despierta verdadero interés entre los estudiantes; además, “[...] el uso de la RA potencia escenarios formativos más motivadores, colaborativos e interactivos y ayuda a una educación más abierta y creativa”. (p. 32).
En contra parte, Cubillo et al. (2014) consideran, la RA como una herramienta al servicio de la educación, como una tecnología con características propias, que la mera visualización de los recursos virtuales deja incompleta la experiencia de aprendizaje y, por ende, es necesario contextualizar y apoyar de forma directa o indirecta por el profesor para obtener una experiencia educativa de calidad. Maquilón, Mirete \& Avilés (2017, p. 197) Aplicar un recurso innovador suele conllevar un cierto esfuerzo formativo. Si además se trata de una tecnología emergente, que está aún en desarrollo, este factor puede hacer al docente no considerar la RA como un recurso plausible. La idea de que para usar la RA son necesarios conocimientos informáticos avanzados o una formación tediosa en tecnologías aplicadas, puede ser también un motivo de rechazo de la RA, que se pretende modificar con la propuesta didáctica que se presenta a continuación. Así como el uso de una pizarra digital interactiva puede aprenderse por parte del docente en unas pocas horas, el uso de la RA también tiene algunas modalidades de aprendizaje sencillo. Asimismo, con esta propuesta didáctica se intenta evaluar el nivel de competencia digital del alumnado de secundaria y comprobar si el aprendizaje del uso de la RA es un proyecto de aprendizaje realizable en un tiempo razonable.

Cabero \& Barroso (2018) "los alumnos muestran altos niveles de satisfacción cuando participan en experiencias de este tipo, y ello es independiente del nivel de estudios en el cual se realice la experiencia, así como de los contenidos curriculares sobre los que versaba." (p. 9). Alvarez-Marin et al., (2017) presentan algunas apreciaciones de los estudiantes sobre la tecnología de la RA permiten visualizar de manera completa el problema; la apreciación final es que esta tecnología acerca al estudiante a una comprensión visual apropiada con respecto a la problemática presentada, debido a que la forma tradicional en la que se plantean estos ejercicios existen aspectos que no se pueden visualizar fácilmente; por lo que la aplicación con RA busca que "[...] impacte positivamente en la capacidad que tengan los estudiantes en la resolución de ejercicios de la temática, tomando en consideración la oportunidad que tienen de visualizar elementos que apoyen su desarrollo y capacidad de razonamiento" (p. 41). 
A pesar de los beneficios que ofrece la RA en la enseñanza, no se puede perder de vista que la tecnología en si no produce cambios, es necesario antes reflexionar sobre si debe utilizarse (Martínez \& Fernández, 2018).

De igual manera, Martins et al. (2019) indican que el crecimiento desmedido de información en Internet ha generado problemas para encontrar recursos pedagógicos de calidad. Cabero-Almenara, Vázquez-Cano \& LópezMeneses (2018, p. 32) señalan que para realizar una adecuada implementación, existen una serie de inconvenientes, algunos de ellos son: el requerimiento de inversión económica que garantice el acceso a esta tecnología en las aulas y centros formativos, también es necesario contar con una formación específica para manipular la tecnología y proponer actividades formativas, del mismo modo, el uso de la RA se asocia con un incremento del distanciamiento humano, poco asertiva que deshumaniza los procesos de enseñanzaaprendizaje. Finalmente, muchos de los sistemas no se adaptan a las diferentes necesidades especiales que puedan tener los estudiantes en las aulas.

Existe gran cantidad de aplicaciones, Reinoso (2012, citado por Prendes, 2015) identifica seis tipos de aplicaciones significativas de RA en la educación, agrupándolas en: (i) aprendizaje basado en el descubrimiento, (ii) desarrollo de habilidades profesionales, (iii) juegos educativos con RA, (iv) modelado de objetos $3 \mathrm{D}$, (v) libros con RA y (vi) materiales didácticos. La investigación hace alusión a la última categoría, se planteó como objetivo general desarrollar un material didáctico con RA de la asignatura de Mercado y Consumo, para alumnos de la licenciatura en Diseño Industrial del Centro Universitario UAEM Valle de Chalco.

\section{Metodología}

En el estudio se examina (i) la necesidad y desarrollo de una aplicación con realidad aumentada que contribuya en la asignatura de mercado y consumo y (ii) la percepción de los docentes y alumnos sobre la calidad de la aplicación móvil con RA.

Procedimiento
El estudio consistió en tres etapas: (i) se establecieron los participantes del estudio, se seleccionaron los aspectos a incluir en la aplicación y se desarrollaron y seleccionaron los instrumentos que se utilizarían para determinar el beneficio del uso de la RA y la percepción u opinión de los docentes y alumnos sobre la aplicación con realidad aumentada.

\section{Participantes.}

El muestreo aleatorio fue el método utilizado para el presente estudio. Con relación a los alumnos la población de LDI es de 263, pero los de $5^{\circ}$. semestre a quienes se les imparte la asignatura de mercado y consumo son 76 , cantidad que se tomó como la población para calcular el tamaño de la muestra, con 95\% de confianza y $5 \%$ de margen de error, obteniéndose el tamaño de 64 estudiantes (51 hombres y 13 mujeres). Y participaron 9 docentes total de integrantes de la academia del área curricular Económica Administrativa de LDI (se aplicaron los mismos parámetros de nivel de confianza y margen de error), 6 del género masculino y 3 femenino.

El objetivo de la asignatura de Mercado y consumo es "analizar los factores económicos y de mercado que influyen en la competitividad del Diseño Industrial en el contexto." (UAEMéx, 2015, p. 187).

\section{Instrumentos.}

Se aplicaron tres instrumentos, se inició con una prueba diagnóstica, la cual está integrada por 10 preguntas cerradas para identificar los conocimientos de los alumnos sobre el tema (el diseño industrial en el marco del sector empresarial), el mismo cuestionario se aplicó al final de interactuar con la aplicación.

El segundo, es un instrumento de Martínez \& Fernández (2018) se compone de 23 ítems sobre la realización de objetos de realidad aumentada, se adecuó a 15 preguntas que aplican al material desarrollado, el instrumento se presenta en escala Likert 1 (totalmente en desacuerdo) a 4 (totalmente de acuerdo). 
Y el tercero, se determinó utilizar la rúbrica para aplicaciones móviles educativas propuesta por Cebrián (2018) alojada en Corubic, está compuesta de cinco aspectos: el diseño $10 \%$, manejabilidad $10 \%$, velocidad de proceso $20 \%$, calidad el $30 \%$ y utilidad $30 \%$ (para un $100 \%$ ) cada una con cuatro respuestas en escala Likert. (ii) Consistió en el desarrollo de la aplicación con realidad aumenta utilizando el software unity, participaron en la elaboración cuatro docentes: diseñadora gráfica, informática, pedagoga, y dos profesores de la materia en cuestión, por el momento se encuentra alojada en el repositorio del Cuerpo Académico de manera local, está en revisión en el Sistema para Evaluar la Calidad de los Medios Educativos (SECME) y cuando sea aprobada se coloque en el repositorio institucional (RI) de la Universidad Autónoma del Estado de México de manera abierta en enero de 2020. Cabe mencionar que se realizaron dos guías de usuario que incluye las instrucciones para el docente y los estudiantes sobre el uso de la aplicación; en dicha guía se describen diferentes aspectos, que van desde los objetivos que se persiguen, ubicación de la aplicación para descarga, recomendaciones para el uso del recurso.

(iii) Fue la incorporación de la aplicación en el aula, se inició con la evaluación diagnóstica que se contestó en línea, después viene la intervención docente y uso de la aplicación, posteriormente, se aplicó el ejercicio del tema; las encuestas para conocer la opinión sobre la aplicación fueron también en línea a través de google forms, los docentes tuvieron 5 días para responder y los alumnos una semana ambos durante el mes de mayo de 2019. Se continuó con el proceso de análisis de los datos, para finalizar con la presentación de resultados.

\section{Resultados}

Se presentan en dos secciones: (i) La aplicación con RA y 2) los resultados obtenidos de los instrumentos aplicados.

(i) La aplicación con RA, se incorporaron cuatro aspectos del tema: El diseño industrial y su participación en los sectores económicos. Una vez que se ha descargado y ejecutado la aplicación se visualiza la pantalla de inicio se muestra en la figura 1 la interfaz.

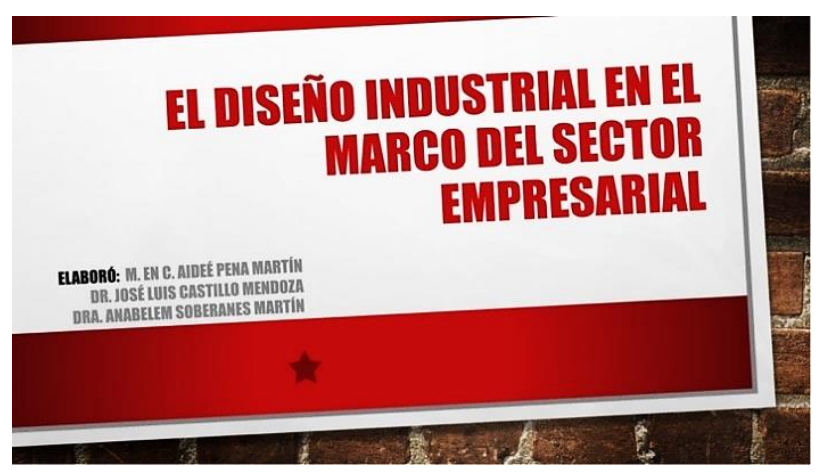

Figura 1 Pantalla de inicio

Fuente: elaboración propia

La siguiente pantalla es del menú se muestra en la figura 2 el contenido de la aplicación son los subtemas: 1) Sectores empresariales, 2) Clasificación de los sectores, 3) Clasificación de las empresas y 4) Ciclo de vida de la empresa.

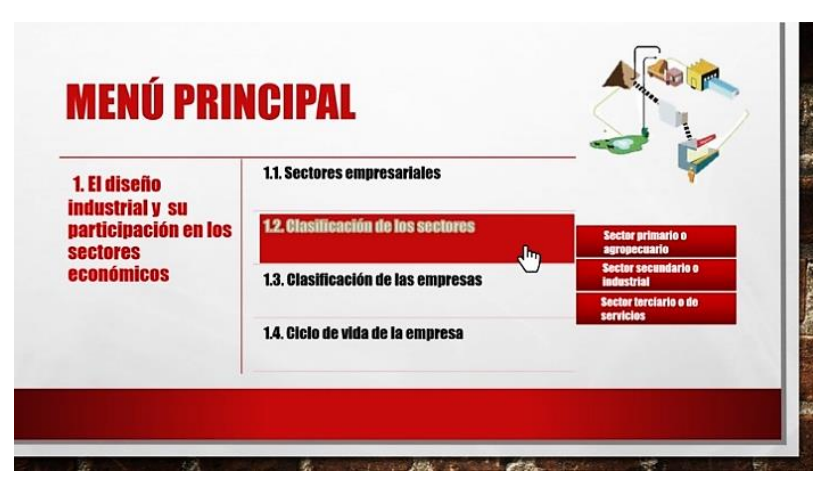

Figura 2 Menú principal

Fuente: elaboración propia

La pantalla que se presenta en la figura 3 presenta la clasificación de las empresas de acuerdo con su actividad o giro. En la figura 4, se presenta el contenido del sector primario o agropecuario y la reproducción de un video sobre el tema. La figura 5 presenta a una alumna visualizando un video sobre las empresas comerciales.
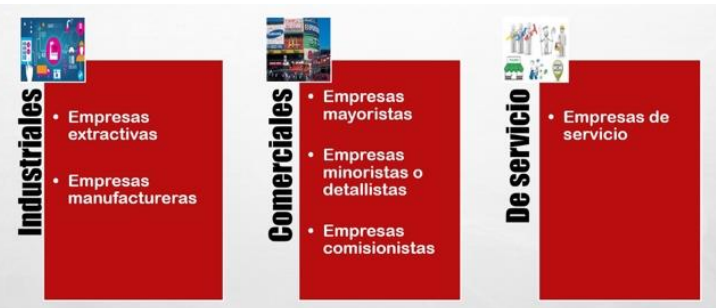

BLASIFIBACIÓN DE LAS EMPRESAS DEAGUERDO GON SUAGTUDDMO GIRO

Figura 3 Pantalla clasificación de las empresas Fuente: elaboración propia 


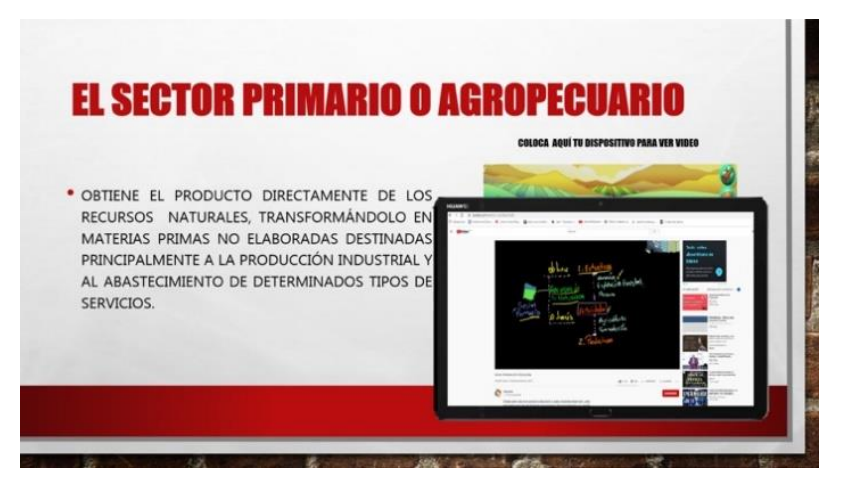

Figura 4 Pantalla del sector primario Fuente: elaboración propia

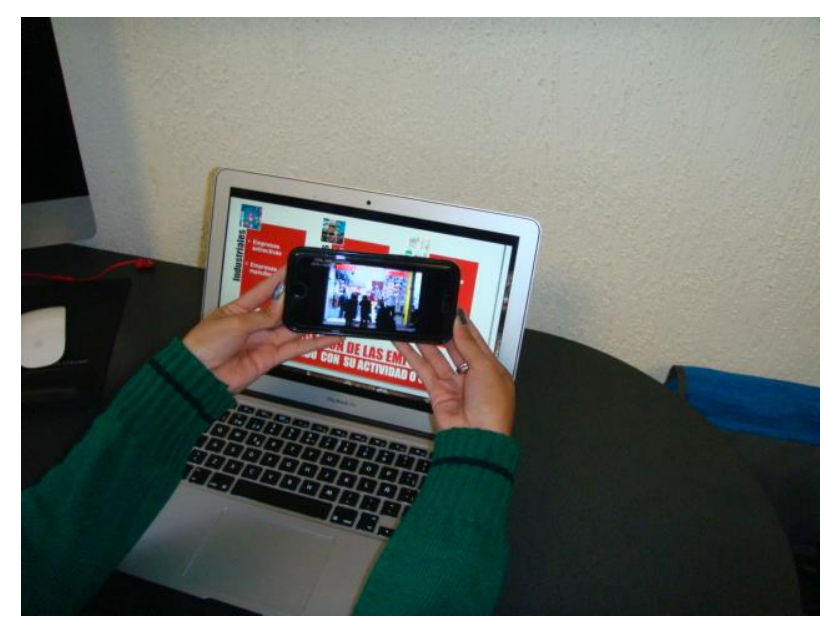

Figura 5 Alumna visualizando video Fuente: elaboración propia

Los resultados del cuestionario diagnóstico que se aplicó antes de utilizar la aplicación, los alumnos contestaron de manera correcta $41.1 \%$, incorrecta $35.8 \%$ y no contestaron $23.1 \%$, el gráfico 1 muestra la información por cada pregunta.

\section{Cuestionario Inicial}

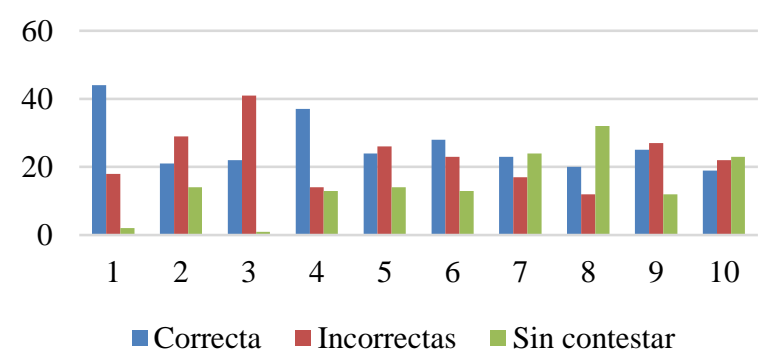

Gráfico 1 Cuestionario diagnóstico

Fuente: elaboración propia

Y los resultados del cuestionario final, los estudiantes mejoraron notablemente al contestar correctamente el $95.47 \%$, incorrecta $2.81 \%$ y no contesto solo el $1.71 \%$. El gráfico 2 , presenta los resultados por pregunta.

\section{Cuestionario Final}

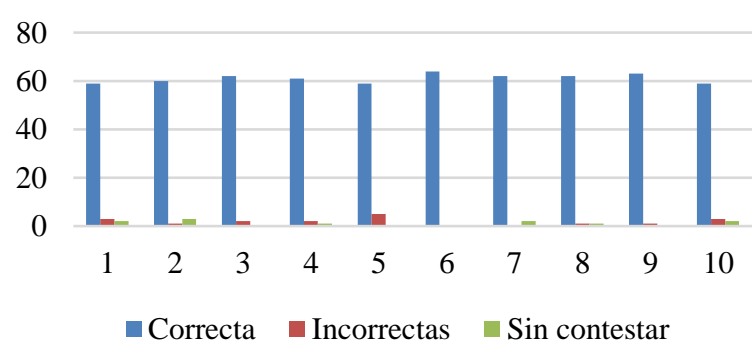

Gráfico 2 Cuestionario de cierre

Fuente: elaboración propia

(iii) Los resultados de las encuestas de opinión de los estudiantes se presentan a continuación, la primera pregunta se refiere si el alumno encuentra a la RA útil en su aprendizaje, a lo cual los estudiantes contestaron $8(13 \%)$ que totalmente de acuerdo y $56(87 \%)$ que, de acuerdo, y ninguno que en desacuerdo o totalmente en desacuerdo, lo que permite identificar la utilidad que identifican los alumnos de la RA (ver gráfico 3).

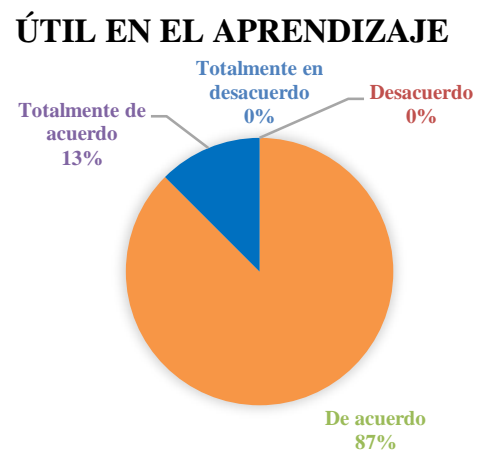

Gráfico 3 Utilidad para el aprendizaje

Fuente: elaboración propia

En relación con la percepción que tienen si la RA les permite adquirir conocimientos de manera más rápida, $26(40.6 \%)$ respondieron estar totalmente de acuerdo, $36(56.2 \%)$ y 2 $(3.1 \%)$ en desacuerdo, lo que permite identificar la facilidad que consideran para aprender al usar RA. Lo cual se ratifica con las respuestas sobre si el uso de la RA es sencillo y claro, a lo que contestaron $32(50 \%)$ que totalmente de acuerdo, $31(48.4 \%)$ que de acuerdo y solo uno (1.6\%) que en desacuerdo, además, si encontraban la RA fácil de usar, 38 (59.4\%) estudiantes expresaron estar totalmente de acuerdo, 25 (39.1\%) de acuerdo y uno (1.7\%) en desacuerdo; y si el aprendizaje del uso de la RA ha sido fácil, los dicentes respondieron $29(29.7 \%)$ totalmente de acuerdo y $45(70.3 \%)$ de acuerdo. 
Con respecto, si considera que el uso de la RA para el aprendizaje es una buena idea, 23 contestaron totalmente de acuerdo, 38 de acuerdo y 3 en desacuerdo (ver gráfico 4).

\section{USO DE LA RA EN EL APRENDIZAJE}

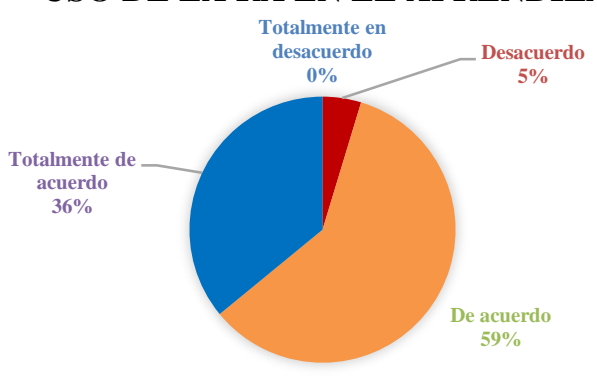

Gráfico 4 Buena idea de uso de la RA en el aprendizaje Fuente: elaboración propia

Otro cuestionamiento, fue si RA hace el aprendizaje más interesante, a lo que los estudiantes respondieron 12 totalmente de acuerdo y 52 de acuerdo (gráfico 5), lo que demuestra que $100 \%$ considera la RA atractiva para su aprendizaje.

\section{RA ES INTERESANTE EN EL} APRENDIZAJE

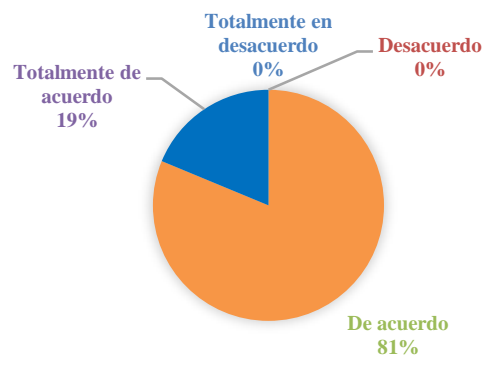

Gráfico $5 \mathrm{El}$ aprendizaje más interesante con RA Fuente: elaboración propia

Si el uso de la RA para el aprendizaje les resulta divertido, los alumnos expresaron 14 (21.9\%) estar totalmente de acuerdo y 50 (78.1\%) de acuerdo, lo que manifiesta que para ningún alumno es aburrido.

Respecto a si les gusta utiliza la RA como herramienta para el estudio los universitarios opinaron 19 estar totalmente de acuerdo, 41 de acuerdo y 4 en desacuerdo (gráfico 6). En cuanto si el profesorado ayuda para el uso de la RA en su aprendizaje, los estudiantes señalaron $15 \quad(23.4 \%)$ que totalmente de acuerdo, 39 (61\%) que de acuerdo y $10(15.6 \%)$ que, en desacuerdo, los alumnos señalan que con la guía de uso y por las características de la RA, es menor el apoyo que se requiere de los docentes.

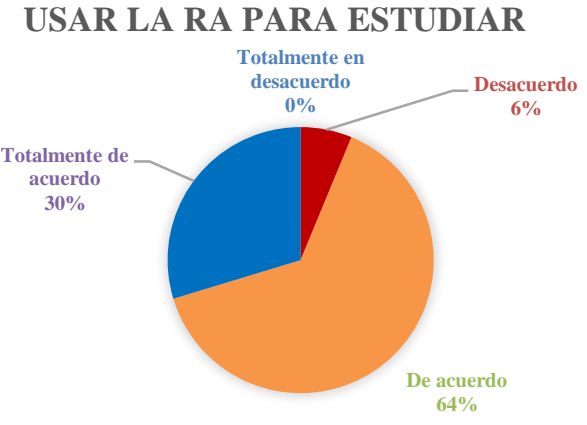

Gráfico 6 La RA como herramienta de estudio Fuente: elaboración propia

También se les preguntó a los dicentes, si tienen los recursos necesarios para utilizar la RA en su aprendizaje, opinaron 60 (93.2\%) que totalmente de acuerdo y 4 (6.2\%) que, de acuerdo, lo cual ratifica lo reportado por Lagunes-Domínguez et al. (2017) sobre el porcentaje de alumnos que cuentan con los recursos necesarios. Respecto a tener el conocimiento necesario para manipular la RA en su aprendizaje, los alumnos consideraron 57 $(89.1 \%)$ que totalmente de acuerdo y 7 (10.9\%) que de acuerdo. Además, se les cuestionó si se sienten insegura/o al utilizar las aplicaciones de $\mathrm{RA}$, a lo que respondieron $15(23.4 \%)$ de acuerdo, $5(7.8 \%)$ en desacuerdo y $44(68.8 \%)$ totalmente en desacuerdo (gráfico 7), se identifica la seguridad de estudiantes al utilizar la aplicación.

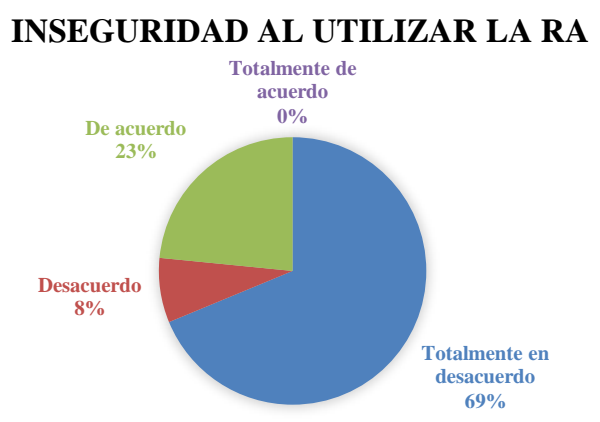

Gráfico 7 Inseguridad del uso de la aplicación Fuente: elaboración propia

Sobre la intención de utilizar las Apps para en su formación, los universitarios 18 (28.1\%) totalmente de acuerdo, $42(62.6 \%)$ de acuerdo y $4(6.2 \%)$ en desacuerdo. Finalmente, si los alumnos recomendasen el uso de las aplicaciones para el aprendizaje, $12(18.8 \%)$ totalmente de acuerdo, $50(78.1 \%)$ de acuerdo y $2(3.1 \%)$ en desacuerdo (gráfico 8$)$. 


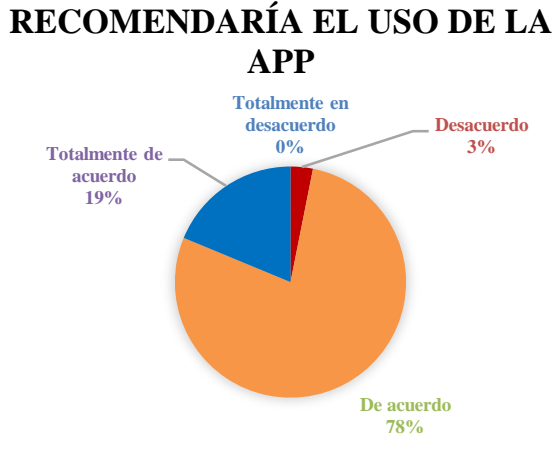

Gráfico 8 Recomendación de uso

Fuente: elaboración propia

La rúbrica de la calidad de la aplicación con RA se conforma de cinco aspectos: el diseño, manejabilidad, velocidad de proceso, calidad y utilidad. Los resultados de los docentes, en cuestión de diseño resalta que $92 \%$ de los docentes consideran que tiene un diseño moderno e innovador, muy atractivo para el público para la que está diseñada y $8 \%$ indicó que tiene un buen diseño destaca por encima de otras aplicaciones de su estilo.

La manejabilidad, 95\% indica que es fácil de utilizar e intuitiva, perfecta para el público a la que está destinada y $5 \%$ señaló que es de fácil manejo, aunque podría presentar dudas si no se utiliza en el período educativo adecuado y $3 \%$ expresó que, aunque útil, se muestra de difícil manejo, se necesitan competencias específicas para su uso y para poder entenderla.

La velocidad de proceso, los docentes expresaron 95\% que los cambios de pantalla y los procesos en general son rápidos y $5 \%$ que se tiene una buena velocidad de proceso.

La calidad, expresaron $95 \%$ de los maestros que goza de una calidad inmejorable educativamente hablando, buen lenguaje, didáctica e instructiva y $5 \%$ que es de buena calidad, aunque presenta ciertas carencias didácticas y/o educativas.

En último lugar, sobre con la utilidad 99\% expresaron que el uso puede ser más que recomendado en el aula para complementar los aprendizajes y $1 \%$ indicó que su uso aporta ciertos conocimientos al alumno y ayuda al profesor en sus explicaciones, pero no lo complementa. Los resultados se comparan con los porcentajes que establece la rúbrica para cada categoría, se calculó el total sumando $94.7 \%$ (ver tabla 1 ).

\begin{tabular}{|l|r|r|}
\hline \multicolumn{1}{|c}{ Categoría } & \% rúbrica & \% obtenido \\
\hline Diseño & 10 & 9.2 \\
\hline Manejabilidad & 10 & 9.5 \\
\hline Velocidad de proceso & 20 & 19.0 \\
\hline Calidad & 30 & 27.3 \\
\hline Utilidad & 30 & 29.7 \\
\hline Total & $\mathbf{1 0 0}$ & $\mathbf{9 4 . 7}$ \\
\hline
\end{tabular}

Tabla 1 Resultados de los docentes

Fuente: elaboración propia

Los resultados de los alumnos son: el diseño resalta que $98 \%$ de los estudiantes consideran que tiene un diseño moderno e innovador, muy atractivo para quien está diseñada la aplicación y $2 \%$ expresó que tiene un buen diseño destaca por encima de otras aplicaciones de su estilo. De la manejabilidad, 99\% opinó que es fácil de utilizar e intuitiva, perfecta para quien está destinada y $1 \%$ señaló que es de fácil manejo, aunque podría presentar dudas si no se utiliza en el período educativo adecuado. Otro aspecto es la velocidad de proceso, expresaron $100 \%$ de los alumnos que los cambios de pantalla y los procesos en general son rápidos. Con respecto a la calidad, manifestaron $99 \%$ que goza de una calidad inmejorable educativamente hablando, buen lenguaje, didáctica e instructiva. En términos de la utilidad, $100 \%$ expresó que el uso puede ser más que recomendado en el aula para complementar los aprendizajes de los alumnos y complementa a su vez las explicaciones del profesor. De manera global se obtiene $99.6 \%$ por parte de los estudiantes sobre la aplicación, después de compararlo con los valores asignados por la rúbrica (tabla 2).

\begin{tabular}{|l|r|r|}
\hline \multicolumn{1}{|c|}{ Categoría } & \% rúbrica & \% obtenido \\
\hline Diseño & 10 & 9.8 \\
\hline Manejabilidad & 10 & 9.9 \\
\hline Velocidad de proceso & 20 & 19.6 \\
\hline Calidad & 30 & 29.7 \\
\hline Utilidad & 30 & 30.0 \\
\hline Total & 100 & 99.6 \\
\hline
\end{tabular}

Tabla 2 Resultados de los alumnos

Fuente: elaboración propia

Con respecto a la rúbrica para aplicaciones móviles educativas en promedio por parte de los docentes se obtuvo $94.7 \%$ y de los alumnos $99.6 \%$. 


\section{Conclusiones}

La aplicación permite explorar el tema del diseño industrial en el marco del sector empresarial, que les causa confusión a los alumnos para identificar el tipo de empresa, las características y sectores; por lo que la aplicación gradualmente permite ir explorando el tema hasta poder aplicarlo para sus propuestas industriales, aprovechando su interés por los recursos digitales y la tendencia a seguir un ritmo de aprendizaje. Los porcentajes obtenidos de los cuestionarios, demuestra que la aplicación es aceptada por los alumnos y docentes al enriquecer el proceso de aprendizaje, se identifica que los contenidos virtuales si contribuyen en la formación de los alumnos de LDI, como lo indican Castillo et al. (2016b) " [...] utilicen las TIC para adquirir o fortalecer el conocimiento mediante la interacción de aplicaciones multimedia, como son vídeos, imágenes, animaciones y texto; haciendo que la adquisición del conocimiento sea más activa [...]" (p. 96). De igual manera, lo expresan Martínez \& Fernández (2018) al considerar la RA como una tecnología que mejora el aprendizaje en los contextos de la educación superior.

Se reconoce que a parte de los conocimientos disciplinares adquiridos por el uso de la aplicación con RA promueve otros aspectos como lo indican diversos autores (Martínez \& Fernández, 2018; Blas et al., 2019) promueve la motivación, la curiosidad y la creatividad, potenciando, la adquisición de competencias necesarias para el futuro profesional. Aunque fue posible determinar una mejora notable entre los resultados de los cuestionarios sobre el tema, no se podría asegurar que exista una relación entre la motivación de los estudiantes y el rendimiento que lograron.

Lo que se puede determinar es la aprobación de los materiales didácticos con RA por parte de los docentes, sin embargo, como lo expresan Cupitra-García \& Duque-Bedoya (2018) “[...] estos desarrollos tecnológicos traen inmerso el reto para que el docente conciba usos didácticos de dispositivos móviles para mejorar el proceso de enseñanza aprendizaje." (p. 252), lo importante es la incorporación sin caer en excesos y recordando que son un apoyo al docente.
De igual manera, deben considerarse limitaciones, como la infraestructura tecnológica de la institución, que a veces limita el diseño instruccional del docente y el trabajo colaborativo considerado en el aprendizaje con uso de tecnología. Como trabajo futuro se está adecuando el material de acuerdo con las observaciones, para el próximo semestre para después usar un grupo control para comparar el impacto del uso de la aplicación con RA en la asignatura de mercado y consumo, así como diseñar y validar los instrumentos que permitan determinar la existencia significativa entre rendimiento, motivación y la calidad de la aplicación.

\section{Referencias}

Alvarez-Marin, A., Castillo-Vergara, M., Pizarro-Guerrero, J. \& Espinoza-Vera, E. (2017). Realidad Aumentada como Apoyo a la Formación de Ingenieros Industriales. Formación Universitaria, 10 (2):31-42.

Arteaga, I.G. \& Pino, C.W. (2018): La realidad aumentada en entornos educativos. Revista Atlante: Cuadernos de Educación y Desarrollo. En línea: https://www.eumed.net/rev/atlante/2018/09/real idad-aumentada-educativos.html

Badilla, M. \& Sandoval, A.M. (2015). Realidad aumentada como tecnología aplicada a la educación superior: Una experiencia en desarrollo. Innovaciones educativas, XVII (23): 41-49.

Bernal, L., \& Ballesteros-Ricaurte, J. (2017). Metodología para la construcción de objetos virtuales de aprendizaje, apoyada en realidad aumentada. Sophia, 13 (1): 4-12.

Blas, D., Vázquez-Cano, E., Morales, M.B. \& López, E. (2019). Uso de apps de realidad aumentada en las aulas universitarias. Campus Virtuales, 8(1): 37-48.

Cabero-Almenara, J., De la Horra, I, \& Sánchez, J. (2018). La realidad aumentada como herramienta educativa. Ediciones Paraninfo, S.A. 
Cabero, J., \& Barroso, J. M. (2018). Los escenarios tecnológicos en Realidad Aumentada (RA): posibilidades educativas en estudios universitarios. Aula abierta, 47(3): 327-336.

Cabero-Almenara, J., Vázquez-Cano, E., \& López-Meneses, E. (2018). Uso de la realidad aumentada como recurso didáctico en la enseñanza universitaria. Formación universitaria, 11(1): 25-34.

Cabero, J., Barroso, J., \& Llorente, C. (2019). La realidad aumentada en la enseñanza universitaria. REDU. Revista de Docencia Universitaria, 17(1): 105-118.

Castillo, J. L., Martínez, M., Soberanes, A. \& Sánchez, J. M. (2016a). Definición de estilos de aprendizaje en estudiantes universitarios de acuerdo con la programación neurolingüística. Docencia e Investigación Educativa, 2(3): 2534.

Castillo, J. L., Soberanes, A., Peña, A. \& Martínez, M. (2016b). Proceso de aprendizaje en la materia de envase y embalaje del diseñador industrial. Pistas Educativas, 122(CITEC 2016): 86-99.

Cebrián, D. (2018). Rúbrica para Aplicaciones móviles educativas. Disponible en: https://corubric.com/index.php?r=publicrubric\%2Fview\&id=491

Cheng, K.H. (2017). Reading an augmented reality book: An exploration of learner's cognitive load, motivation and attitudes. Australasian Journal of Educacional Technology, 33(4): 53-69.

Cubillo, J.; Martín, S.; Castro, M. \& Colmenar, A. (2014). Recursos digitales autónomos mediante realidad aumentada. RIED. Revista Iberoamericana de Educación a Distancia, 17(2): 241-274.

Cupitra-García, A., \& Duque-Bedoya, E. (2018). Profesores aumentados en el contexto de la realidad aumentada: una reflexión sobre su uso pedagógico. El Ágora USB, 18 (1): 245255. http://dx.doi.org/10.21500/16578031.3178 Díaz-Campos, B. (2016). Realidad aumentada en la educación. Revista entorno, (61): 47-53.
Fombona, J., Pascual-Sevillano, M.A. \& González-Videgaray, M.C. (2017). M-learning y realidad aumentada: Revisión de literatura científica en el repositorio WoS. Comunicar, XXV (52): 63-72.

Harley, J., Poitras, E., Jarrell, A., Duffy, M., \& Lajoie, S. (2016). Comparing Virtual and Location-Based Augmented Reality Mobile Learning: Emotions and Learning Outcomes. Educational Technology Research and Development, $\quad 64(3)$ : 359-388. https://doi.org/10.1007/s11423-015-9420-7

Lagunes-Domínguez, A., Torres-Gastelú, C.A, Angulo-Armenta, J., \& Martínez-Olea, M.Á. (2017). Exploration toward Mobile Learning in University Students. Formación universitaria, 10(1): 101-108. doi: https://dx.doi.org/10.4067/S0718-

50062017000100011

León, F., Duque, E., \& Escobar, P. (2018). Estrategias de formulación de preguntas de calidad mediadas por realidad aumentada para el fortalecimiento del pensamiento científico. Revista Mexicana de Investigación Educativa, 23(78): 791-815.

Maquilón, J.J., Mirete, A.B. \& Avilés, M. (2017). La Realidad Aumentada (RA). Recursos y propuestas para la innovación educativa. Revista Electrónica Interuniversitaria de Formación del Profesorado, 20(2): 183-203. DOI: http://dx.doi.org/10.6018/reifop.20.1.290971

Martins, A., Fracchia, C.C., Allan, C., Parra, S., Baeza, N., Celeste, C., ... \& Laurent, R. (2019). Desarrollo de herramientas informáticas y sus aplicaciones en el ámbito educativo. In XXI Workshop de Investigadores en Ciencias de la Computación (WICC 2019, Universidad Nacional de San Juan).

Martínez, S. \& Fernández, B. (2018, julio). Objetos de Realidad Aumentada: Percepciones del alumnado de pedagogía. Píxel-Bit. Revista de Medios y Educación, (53): 207-220. doi: http://dx.doi.org/10.12795/pixelbit.2018.i53.14 Prendes, C. (2015). Realidad aumentada y educación: análisis de experiencias prácticas Píxel-Bit. Revista de Medios y Educación, 46: 187-203. 
Tecnológico de Monterrey (2017). Reporte Edu Trends. Realidad Aumentada y Realidad Virtual, Monterrey: Tecnológico de Monterrey. UAEMéx. (2015). Plan de estudios de Diseño Industrial. Toluca, México: UAEMex.

Villalustre, L., \& Del Moral, M. (2018). Geolocalización y realidad aumentada para un aprendizaje ubicuo en la formación inicial del profesorado. @tic. Revista d'innovació educativa, (21): 40-48. http://dx.doi.org/https://doi.org/10.7203/attic.21.12 633 